\title{
Performance of the parametric receiving array: Effects of misalignment
}

\author{
Kenneth G. Foote \\ Institute of Marine Research, 5011 Bergen, Norway \\ Jacqueline Naze Tjøtta and Sigve Tjøtta \\ Department of Mathematics, The University of Bergen, 5014 Bergen, Norway
}

(Received 14 October 1986; accepted for publication 17 June 1987)

\begin{abstract}
The difference frequency sound field from two concentric but misaligned, axisymmetric, planar transducers in a nondissipative and nondispersive medium is developed as a special case of the general theory [Garrett et al., J. Acoust. Soc. Am. 75, 769-779 (1984)]. Effects of misalignment of pump, source, and hydrophone on the performance of the parametric receiving array are quantified in numerical examples. These include the effect of interaction in the nearfields of both pump and source transducers. The results show that the best performance is obtained for good alignment, high pump frequency, and placement of the hydrophone within or not far from the source nearfield.
\end{abstract}

PACS numbers: 43.25.Lj, 43.30.Qd

\section{INTRODUCTION}

The parametric receiving array consists of two acoustic elements and some electronics. ${ }^{1,2}$ One of the elements, called the pump, is active. It transmits sound, of known directional and spectral character, which, through its nonlinear interaction with another, unknown sound field, due to the so-called source, results in the generation of a third sound field. This nonlinearly, or parametrically, generated sound field is detected by the other, passive element, or hydrophone.

No matter what is known about the source field, the alignment of source and pump fields is, in general, only approximate. Similarly, the alignment of hydrophone and pump is generally imperfect. Problems with alignment may be particularly acute for the kind of application envisaged by Chotiros. ${ }^{3}$ In this, the noise radiated by a vessel underway is measured through its parametric interaction with the highfrequency sound field from an ad hoc vessel-borne pump transducer. The nonlinearly generated sound is detected by a remote hydrophone. The approximate direction of the vessel noise is outwards, but the precise direction is undoubtedly both frequency and speed dependent. Thus there may not be a definite condition of source-pump alignment that applies either to a single frequency at all vessel speeds or to the band of radiated frequencies at a single speed. Positioning of the hydrophone in the narrow beam of the pump transducer is fraught with its own error sources, e.g., those due to vessel movement, ambient noise, and sea state.

Thus the condition of optimal performance: perfect alignment of pump, source, and hydrophone, is highly unlikely. But what is the precise effect of misalignment on the performance of the parametric receiving array? That is the question to be addressed here.

Most previous studies, in one way or another, have neglected diffraction in describing the noncollinear interaction of pump and source waves. Examples include the popular models of a planar source wave and a pump wave that is either collimated ${ }^{4,5}$ or spherical. ${ }^{6,7}$ Neither model can de- scribe the parametric fields produced in the nearfield of the source or relate the farfield solution to the acoustic field on the source. Rolleigh, however, has noted the importance of accounting for diffraction when the hydrophone is in the interaction region and the primary beams are not collimated. $^{8}$

Hamilton et $a l^{2}$ have contributed to the general diffraction solution by considering the noncollinear interaction of pump and source fields from circular transducers with Gaussian amplitude shading and linear phase shading. The hydrophone is assumed to be aligned with the pump.

In this study, the pump and source are again represented by circular transducers, but with independent Gaussian or uniform amplitude shading. The pump transducer is assumed to be physically steered, although this could also be accomplished by linear phase shading without loss of generality. In addition to studying some of the same effects considered by Hamilton et al., this study treats the effect of misalignment of hydrophone and pump.

The plan of the article is simple. The approximate formula for the noncollinear interaction of two monochromatic sound fields due to concentric, axisymmetric, planar transducers in a nondissipative and nondispersive medium is drawn from the general theory, ${ }^{9}$ applicable in the quasilinear and parabolic approximations. Numerical results are then presented for several effects of misalignment. These are discussed critically before summary statement of the general findings.

\section{THEORY}

The general theory applied here, as expressed in Ref. 9, Eq. (8), depends on two fundamental assumptions. The first is the quasilinear approximation. This assumes that the several interacting waves are relatively weak, which allows simple substitution of the primary fields of the pump and source in the nonlinear source term of the governing equation.

The second, parabolic approximation is invoked several 
times in the development of the theory. It is first applied to each of the primary fields. For a planar circular transducer of radius $a$ radiating with the wavenumber $k$, the parabolic approximation allows description of the radiated field in the paraxial region at distances greater than $a(k a)^{1 / 3}$ from the transducer. At lesser distances the application is not guaranteed, but may nonetheless be quite useful, at least for distances that are not very small compared to $a(k a)^{1 / 3}$. This has been demonstrated earlier by the authors's beam pattern computations for focused but linear radiators based on straightforward evaluation of the exact integral expression and use of the parabolic approximation.

The parabolic approximation is also used in describing the acoustic field resulting from the parametric interaction of the primary fields. This assumes that the angle of intersection of the primary fields is not too large.

In addition to these general assumptions, a number of particular assumptions are made in reducing the cited equation. These are that the primary fields are due to concentric, planar, circular transducers and that the medium is nondissipative and nondispersive. Thus the wavenumber $k$ and radius $a$ of each transducer, denoted with the subscripts 1 and 2 for the respective higher-frequency pump and lower-frequency source, are such that $k a \gg 1$. In practice, the lower limit of applicability is about 3 . Additionally, the shading functions of the transducers are axisymmetric. In terms of the radial distance $x$, measured in the plane of the transducer from the transducer center, the amplitude function $q(x)$ of pump and source can be expressed as $q_{1}=q_{1}\left(x_{1}\right)$ and $q_{2}=q_{2}\left(x_{2}\right)$, respectively. Also, the medium is described by just three properties: the static density $\rho_{0}$, small-amplitude sound speed $c_{0}$, and nonlinearity parameter $\beta=1+B / 2 A$. These and other notations in this article follow the usage in Ref. 2.

For the geometry indicated in Fig. 1, the difference frequency pressure field at the hydrophone, assumed extensionless, is $p_{-}(z, \mathbf{x})=\exp \left(i k_{-} z\right) q_{-}(z, \mathbf{x})$, where $k_{-}=k_{1}$ $-k_{2}$ is the difference frequency wavenumber. The amplitude $q_{-}(z, \mathbf{x})$ is derived as a special case of Eq. (8) of Ref. 9, viz.,

$$
\begin{aligned}
q_{-}(z, \mathbf{x})= & \frac{\beta k_{1} k_{2} k_{-}}{4 i \pi \rho_{0} c_{0}^{2} z} \exp \left(i \frac{k_{-} x^{2}}{2 z}\right) \\
& \times \int_{0}^{\infty} \int_{0}^{\infty} \int_{0}^{2 \pi} \exp \left(i \frac{F}{2 k_{-} z}\right) E_{1}\left(i \frac{k_{1} k_{2}}{2 k_{-} z} G\right) \\
& \times J_{0}\left(H^{1 / 2}\right) q_{1}\left(x_{1}\right) q_{2}^{*}\left(x_{2}\right) x_{1} x_{2} d \phi d x_{1} d x_{2},
\end{aligned}
$$

where

$$
\begin{aligned}
H= & (x / z)^{2} F+\left(\eta x_{2}\right)^{2}+2(x / z) \eta x_{2} \\
& \times\left[k_{1} x_{1} \cos (\phi-\epsilon)-k_{2} x_{2} \cos \epsilon\right], \\
F= & \left(k_{1} x_{1}\right)^{2}+\left(k_{2} x_{2}\right)^{2}-2 k_{1} k_{2} x_{1} x_{2} \cos \phi, \\
\eta= & k_{2} \sin \theta, \\
G= & x_{1}^{2}+x_{2}^{2}-2 x_{1} x_{2} \cos \phi .
\end{aligned}
$$

The usual denotations are used for the exponential integral $E_{1}$ and the Bessel function of order zero $J_{0}{ }^{10}$
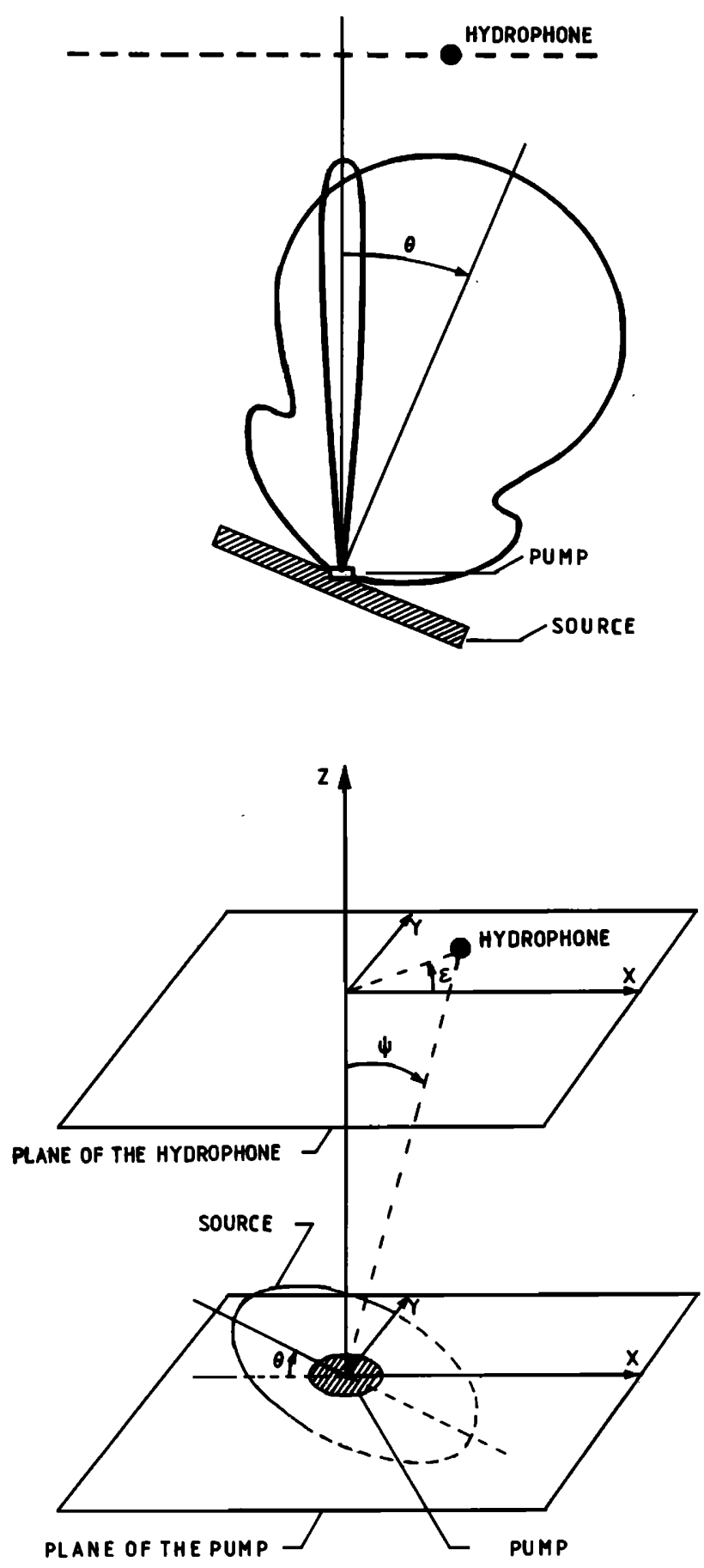

FIG. 1. Geometry.

\section{NUMERICAL RESULTS}

The examined parameter ranges of the present computations are similar to those chosen by Hamilton et al. In particular, $a_{1}=1, a_{2}=20$, and $k_{1}=15$. Distances from the pump are expressed in units of the Rayleigh distance of the source, namely, $k_{2} a_{2}^{2} / 2$. The distance $z$ along the pump axis is thus expressed through the nondimensional quantity $\sigma_{2}=2 z / k_{2} a_{2}^{2}$.

The amplitude shading functions of the pump and source transducers may be uniform or Gaussian. For uniform shading, 


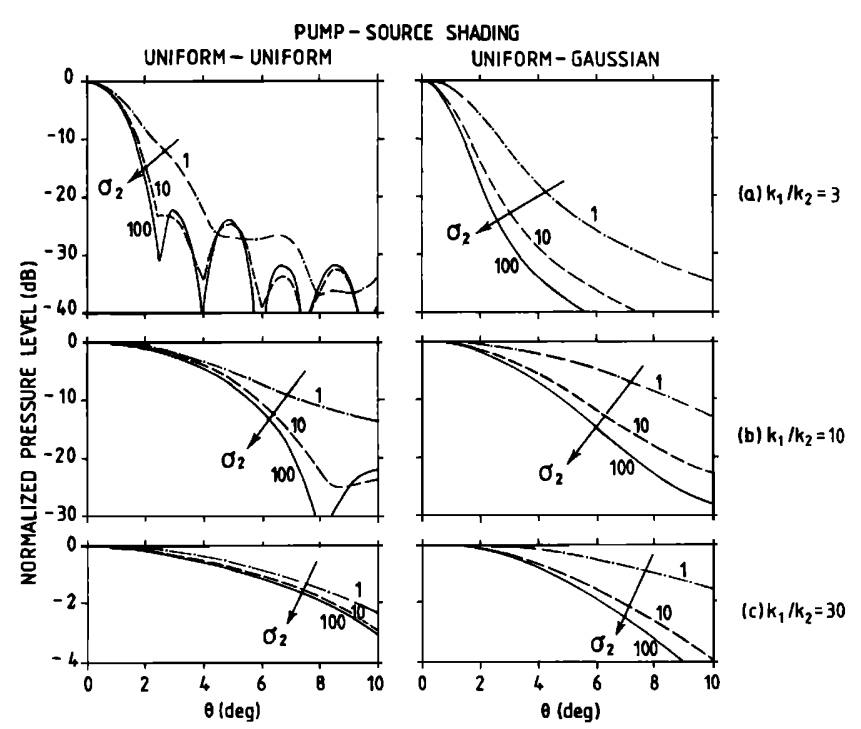

FIG. 2. Dependence of the difference frequency sound-pressure level on the angle of misalignment $\theta$ between the pump and source transducers. The hydrophone, or field point, lies on the pump axis at distance $\sigma_{2}$ from the plane of the pump.

$$
q_{j}\left(x_{j}\right)= \begin{cases}p_{j 0}, & \text { for } x_{j} \leqslant a_{j}, \\ 0, & \text { for } x_{j}>a_{j},\end{cases}
$$

where $p_{j 0}$ is constant, and $j=1$ for the pump transducer and $j=2$ for the source transducer. For Gaussian shading,

$$
q_{j}\left(x_{j}\right)= \begin{cases}p_{j 0} \exp \left(-x_{j}^{2} / a_{j}^{2}\right), & \text { for } x_{j} \leqslant 2.5 a_{j}, \\ 0, & \text { for } x_{j}>2.5 a_{j} .\end{cases}
$$

No phase shading is applied.

Evaluation of Eq. (1) was accomplished in all cases by means of an automatic integration routine developed by Berntsen. ${ }^{11,12}$

Two generic cases of misalignment are studied.

(1) Misalignment of pump and source only. The hydro- phone is assumed to be aligned with the pump; thus $\psi=0$ according to the nomenclature of Fig. 1. The effect of misalignment is first shown, in Fig. 2, through the dependence of the difference frequency sound-pressure level on the angle of misalignment between pump and source for fixed ranges from the pump. The computations are normalized to the respective on-axis, thence peak, values. The effect of misalignment is also shown, in Fig. 3, through the dependence of the difference frequency sound-pressure level on the range along the pump axis for fixed angles of misalignment. Normalization is performed here with respect to the quantity $P_{0}=\beta p_{10} p_{20} / \rho_{0} c_{0}^{2}$.

(2) Misalignment of pump, source, and hydrophone. This effect is quantified, in Fig. 4, by showing the range of variation in the difference frequency sound-pressure level due to uncertainty in azimuthal placement, denoted $\epsilon$ in Fig. 1 , of the hydrophone. The hydrophone is assumed to lie at distance $\sigma_{2}=1$, i.e., at one Rayleigh distance of the source hydrophone, from the plane of the pump. The normalization used in Fig. 4 is identical with that of Fig. 2. Thus the values for $\psi=0$ in Fig. 4 are identical with those for the curves $\sigma_{2}=1$ and corresponding angles $\theta$ in Fig. 2 .

\section{DISCUSSION}

In the numerical results presented in Figs. 2-4, only two of the four possible combinations of pump-source amplitude shading are shown. The reason is that the results for the Gaussian-Gaussian combination were, for the most part, indistinguishable from the uniform-Gaussian combination, and the results for the Gaussian-uniform and uniform-uniform combinations were similarly alike. The greatest, or most noticeable, differences between corresponding pairings occurred at the largest hydrophone displacements from the pump axis, as shown in Fig. 4 for the uniform-uniform and uniform-Gaussian combinations. Thus, for the present parameters, the source transducer determines the performance character of the parametric receiving array.

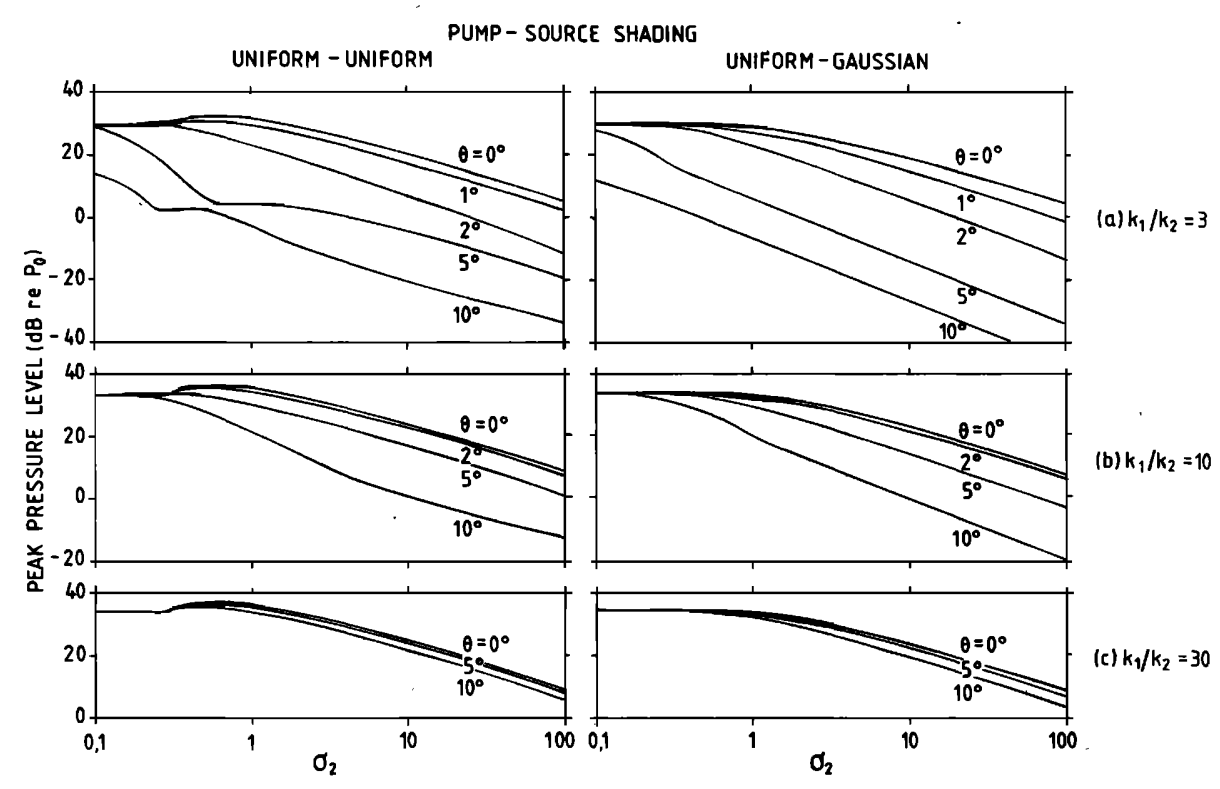

FIG. 3. Range dependence of the difference frequency sound-pressure level due to generally misaligned pump and source transducers. The angle of misalignment is $\theta$. The hydrophone, or field point, is moved along the pump axis, with instantaneous distance $\sigma_{2}$ from the pump transducer. 


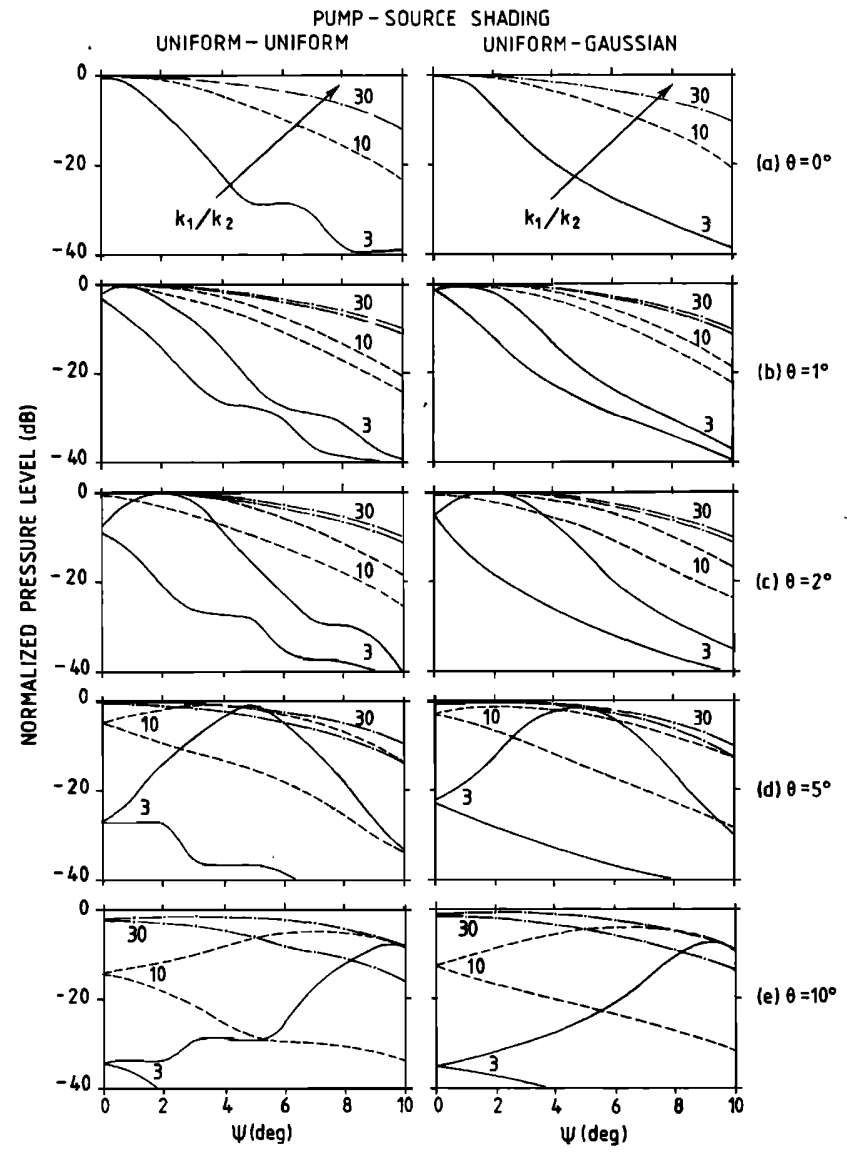

FIG. 4. Range of variation in the difference frequency sound-pressure level, generated by two generally misaligned transducers, due to uncertainty in azimuthal placement $\epsilon$ of the hydrophone at distance $\sigma_{2}=1$ from the plane of the pump. The angle of misalignment of pump and source is $\theta$. The deviation of the hydrophone from the pump axis is $\psi$.

Agreement of the cited computations for the GaussianGaussian pairing with the respective computations by Hamilton et al. in their Figs. 2 and 6 was exact. It is interesting to note that the several numerical methods were independent and quite different. The method of evaluation by Hamilton et al. exploited the fact of Gaussian amplitude shading to derive a simple analytic formula whose most complicated term is an exponential integral with complex argument. The present method involves numerical evaluation of a threedimensional integral. This was developed from the fivefold integration of the general theory in Ref. 9 by the assumptions of axisymmetric, planar pump and source transducers and a nondissipative medium. An advantage of the present approach is the arbitrariness of shading of the pump and source transducer, if axisymmetric.

Neglect of absorption in this work may be remedied in the future. For the case of axisymmetric pump and source transducers, the integration required for evaluating the difference frequency sound-pressure amplitude will then be fourfold. However, the directivity patterns in Figs. 2 and 4 should be changed only slightly. The results in Fig. 3, which show the range dependence of the difference frequency pressure along the pump axis, will, in general, change significantly for typical high pump frequencies. This is because the distance increases from one-tenth to one hundred times the Rayleigh distance of the source transducer. The relative effects of misalignment of pump and source, however, may be expected to remain similar to those in Fig. 3.

As noted in Sec. I, the parabolic approximation was used several times in the development of the general theory. While this is a fundamental limitation, and prevents examination of the effects of misalignment or noncollinearity much outside of the paraxial region, this does not affect the practical conclusions to be drawn from the numerical results. These are (1) that the parametric receiving array performs best when the pump, source, and hydrophone are aligned, (2) that the pump frequency ought to be much higher than the source frequency, and (3) that the hydrophone ought to be located within about one Rayleigh source distance.

The first two, qualitative, conclusions were made by, among others, Berktay in his early study. ${ }^{4}$ Since this was performed on the basis of a quite different model, the robustness of the directivity and frequency condition of the parametric receiving array is apparent. The third, quantitative, conclusion can only be made on the basis of an absolute model which accounts for the detailed nearfield structures of pump and source fields.

\section{CONCLUSION}

Effects of misalignment of pump, source, and hydrophone on the performance of the parametric receiving array have been quantified in the context of a fully diffractive theory. A general formula is presented for the case of axisymmetric pump and source transducers in a nondissipative medium. Numerical examples based on transducers that are uniformly shaded in phase and uniformly or Gaussian shaded in amplitude indicate the importance of (1) alignment of pump, source, and hydrophone, (2) use of a relatively high pump frequency, and (3) placement of the hydrophone within or not much outside the source nearfield.

\section{ACKNOWLEDGMENTS}

The work reported in this article is part of projects supported by The Norwegian Research Council for Sciences and Humanities, and STATOIL, Norway.

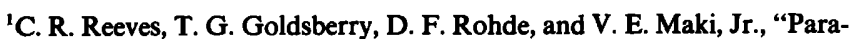
metric acoustic receiving array response to transducer vibration," J. Acoust. Soc. Am. 67, 1495-1501 (1980).

${ }^{2}$ M. F. Hamilton, J. Naze Tjøtta, and S. Tjøtta, "Noncollinear interaction of two sound beams from displaced Gaussian sources, with application to parametric receiving arrays," J. Acoust. Soc. Am. (submitted for publication).

${ }^{3}$ N. P. Chotiros, "Noise measurement in the presence of multipath propagation," Prog. Rep. No. 6, Contract No. AT/2027/0114 ARL, Dept. of Electronic and Electrical Engineering, University of Birmingham, England (1981).

${ }^{4} \mathrm{H}$. O. Berktay, "Parametric amplification by the use of acoustic non-linearities and some possible applications," J. Sound Vib. 2, 462-470 (1965).

${ }^{5}$ P. H. Rogers, A. L. Van Buren, A. O. Williams, Jr., and J. M. Barber, "Parametric detection of low-frequency acoustic waves in the nearfield of an arbitrary directional pump transducer," J. Acoust. Soc. Am. 55, 528534 (1974).

${ }^{6}$ G. R. Barnard, J. G. Willette, J. J. Truchard, and J. A. Shooter, “Paramet- 
ric acoustic receiving array," J. Acoust. Soc. Am. 52, 1437-1441 (1972). ${ }^{7}$ J. J. Truchard, "Parametric acoustic receiving array. I. Theory," J. Acoust. Soc. Am. 58, 1141-1145 (1975).

${ }^{8}$ R. L. Rolleigh, "Difference frequency pressure within the interaction region of a parametric array," J. Acoust. Soc. Am. 58, 964-971 (1975).

${ }^{9} \mathrm{G}$. S. Garrett, J. Naze Tjøtta, and S. Tjøtta, "Nearfield of a large acoustic transducer. Part III: General results," J. Acoust. Soc. Am. 75, 769-779 (1984).
${ }^{10}$ Handbook of Mathematical Functions, edited by M. Abramowitz and I. Stegun (Dover, New York, 1965).

"J. Berntsen, "Cautious adaptive numerical integration over the 3-cube," Reports in Informatics, No. 17, Department of Mathematics, The University of Bergen, Norway (1985).

12J. Berntsen, "User documentation, program CADCUB, a subroutine for numerical evaluation of three-dimensional integrals," Department of Mathematics, The University of Bergen, Norway (1985). 